\title{
Functionalized nanogel for treating activated astrocytes in spinal cord injury
}

\author{
Simonetta Papa ${ }^{\mathrm{a}, 1}$, Valeria Veneruso ${ }^{\mathrm{a}, 1}$, Emanuele Mauri $^{\mathrm{b}, \mathrm{c}}$, Giada Cremonesi ${ }^{\mathrm{b}}$, \\ Xhuljana Mingaj ${ }^{a}$, Alessandro Mariani ${ }^{\mathrm{a}}$, Massimiliano De Paola ${ }^{\mathrm{a}}$, Arianna Rossetti ${ }^{\mathrm{b}}$, \\ Alessandro Sacchetti ${ }^{\mathrm{b}}$, Filippo Rossi ${ }^{\mathrm{b}}$, Gianluigi Forloni ${ }^{\mathrm{a}}$, Pietro Veglianese ${ }^{\mathrm{a}, *}$ \\ ${ }^{a}$ Department of Neuroscience, Istituto di Ricerche Farmacologiche Mario Negri IRCCS, via mario negri 2, 20156 Milano, Italy \\ b Department of Chemistry, Materials and Chemical engineering "Giulio Natta", Politecnico di Milano, via Mancinelli 7, 20131 Milano, Italy \\ ${ }^{\mathrm{c}}$ Department of Engineering, Università Campus Bio-Medico di Roma, via Álvaro del Portillo 21, 00128 Roma, Italy
}

\section{A R T I C L E I N F O}

\section{Keywords:}

Spinal cord injury

Nanogel

Nanoparticles

Astrocytes

Inflammation

\begin{abstract}
A B S T R A C T
Astrogliosis has a unique reaction during spinal cord damage, with helpful or adverse impacts on recovery. There is consequently a pressing need for treatment to target activated astrocytes and their unsafe response after injury to ensure some preservative effect during the progressive damage. We specifically developed and characterized a functionalized nanogel-based nanovector in vitro and in vivo, demonstrating its selectivity towards astrocytes, and limited uptake by macrophages when functionalized with both $\mathrm{NH}_{2}$ and Cy5 groups. In vitro experiments showed that the internalization was mediated by a clathrin-dependent endocytic pathway. After internalization into the cytoplasm of astrocytes, nanogels undergo lysosomal degradation and release compounds with potential therapeutic efficacy.
\end{abstract}

\section{Introduction}

In spinal cord injury (SCI) secondary degenerative events follow the primary damage with progressive cellular death including loss of neurons, astrocytes and oligodendrocytes [1]. A high level of astrogliosis is a common pathological observation, and the pro-inflammatory reaction and dysfunction, with the resulting development of scar tissue and inhibition of regenerative processes of the axons, are considered as the main effects contributing to the progression of the injury. After the trauma astrocytes become responsive and show a range of changes in their morphology and phenotype [2,3]. Reactive astrocytes close to the injury may have effects that are either harmful or benefic in the Central Nervous System (CNS) damage, with variable phenotypes called A1 and A2 $[1,3,4]$. Astrocytes in the A1 state have shown a pro-inflammatory effect, releasing toxic factors for neuronal cells [3,4], while A2 astrocytes have a protective effect by upregulating neurotrophic factors to counteract neuronal degeneration and boost regeneration [3,4]. Genetic ablation of astrocytes and of the resulting scar formation had negative consequences for recovery in SCI [5]. This suggests that treatment for the spinal cord will need to shift astrocytes towards a phenotype better promoting neuronal survival and axonal regeneration instead of reducing the astrocyte-based glial scar.

Different approaches have been employed to neutralize the negative impacts of activated astrocytes [6,7], such as genetic manipulation or pharmacological/biological therapies [6,8-10]. However, these approaches suffer some limitations because they are not selective for astrocytes, or also ethical concerns persist for genetic engineering. Various biomaterials have been developed to deliver pharmacological molecules and/or therapeutic cells in order to improve spinal cord recovery and functional outcomes after injury [11-15]. Polymeric nanoparticles (NPs) offer considerable advantages in compound delivery, controlling their release over time, and can be targeted to specific cells [13,16-19]. Different NPs systems have been examined for treating the injured spinal cord [16], but few attempts have been made to target astrocytes, without selective cellular action [6,20,21]. There is growing evidence that microglia/macrophages are the first cells that process NPs, limiting their achieving other targets. Microglia/macrophages take up foreign materials within a short time, and following this phagocytic mechanism NPs are degraded by the lysosomal system, reducing the therapeutic indications [22]. For a more selectivity, nanovector-mediated NPs are

\footnotetext{
* Corresponding author.

E-mail address: pietro.veglianese@marionegri.it (P. Veglianese).

1 Contributed equally.
} 
needed to reduce phagocytic recognition by microglia/macrophages.

We developed a specific category of nanogels (NG) with high colloidal stability, biocompatibility, and capacity for loading and releasing compounds [23,24]. These NG were investigated for cellular selectivity, uptake, and drug delivery (Rolipram, able to reduce the RNA transcription of pro-inflammatory molecules [25], was used to demonstrate the modulation capability of the system) by identifying the most promising one for selective astrocytes treatment in vitro and in vivo SCI models.

\section{Materials and methods}

\subsection{Materials}

NG used in the experimental procedures required the following polymers: polyethylene glycol (PEG; $\mathrm{M}_{\mathrm{W}}=8 \mathrm{kDa}$, from Merck KGaA, Darmstadt, Germany) and linear polyethylene imine (PEI; $\mathrm{M}_{\mathrm{w}}=2.5$ $\mathrm{kDa}$, from Polysciences Inc., Warrington, USA) used as received, without further purifications. The dyes used to produce fluorescent NG are Cyanine 5-azide (Cy5; $\mathrm{M}_{\mathrm{w}} 1035 \mathrm{Da}$, from Sigma-Aldrich Chemie GmbH, Deisenhofen, Germany) and Rhodamine B base (RhB; $\mathrm{M}_{\mathrm{w}} 479 \mathrm{Da}$, by Sigma-Aldrich Chemie GmbH, Deisenhofen, Germany). RhB was modified to introduce the azide group as described in a previous study [26]. Solvents were of analytical-grade purity. All the rhodamine derivatives were stored at $4{ }^{\circ} \mathrm{C}$, and Cy5 at $-20{ }^{\circ} \mathrm{C}$.

\subsection{NG design and characterization}

\subsubsection{NG synthesis}

NG were synthesized according to experimental procedure already used in previous works $[24,27,28]$ (for synthesis details, see Supplementary data): briefly, PEG hydroxyl groups were modified with imidazole moieties using CDI (hereinafter PEG-CDI), and PEI chains decorated with propargyl groups were further functionalized through the copper-catalyzed azide-alkyne Huisgen cycloaddition (CuAAC) reaction using two dyes: RhB or Cy5, both bearing azide moieties (molar ratio PEI: dye 1:0.025) (Figs. S1-S8) [24,27,28]. Two solutions were prepared separately: the first solution was obtained by dissolving PEGCDI (200 mg, $0.025 \mathrm{mmol}$ ) in $\mathrm{CH}_{2} \mathrm{Cl}_{2}(3 \mathrm{~mL}$ ), and the second one by dissolving in another vessel PEI conjugated with RhB or Cy5 $(0.017$ $\mathrm{mmol}$ ) in distilled water $(5 \mathrm{~mL})$. Under vigorous stirring, the organic solution was added dropwise to the aqueous system, and the final blend was sonicated for $30 \mathrm{~min}$. The polymeric solution was then stirred for 17 $\mathrm{h}$ at $25{ }^{\circ} \mathrm{C}$ (room temperature, r.t.) with the gradual evaporation of $\mathrm{CH}_{2} \mathrm{Cl}_{2}$. The aqueous system was then purified through dialysis, and lyophilized, resulting in a red (NG-RhB) or green (NG-Cy5) solid (Fig. S7).

\subsection{NG coating with 3-bromopropylamine (- $\mathrm{NH}_{2}$ coating)}

The primary amines linkers were grafted around the NG surface already formed, exploiting the unreacted amines in the PEI chains (Figs. S7, S8). NG-RhB and NG-Cy5 (15 mg of NG, corresponding to c.a. $0.566 \mu \mathrm{mol}$ of free $\mathrm{NH}$ groups [24]) were dissolved in distilled water (1 $\mathrm{mL}$ ) and kept under stirring at r.t. with 3-bromopropylamine hydrobromide $\left(5 \mathrm{mg}, 22.64 \mu \mathrm{mol}\right.$ ), the reactant bearing the primary $\mathrm{NH}_{2}$ groups, dissolved in distilled water $(0.5 \mathrm{~mL})$ and added dropwise to the NG solution [24]. The final mixture was then stirred for $17 \mathrm{~h}$ in the dark, at r.t. Using a regenerated cellulose membrane (MW cut-off 6-8 kDa) dialysis against distilled water $(1000 \mathrm{~mL})$ was performed for two days, with daily water exchange, to remove unreacted species and any byproducts. The system was frozen at $-80{ }^{\circ} \mathrm{C}$ and the products were retrieved by lyophilization. These NG coated with primary amine moieties are indicated as $\mathrm{NH}_{2}+\mathrm{RhB} \mathrm{NG}$ and $\mathrm{NH}_{2}+\mathrm{Cy} 5 \mathrm{NG}$.

In vitro or in vivo experiments were done with NG (2.5\%), and Rolipram $(0.7 \mathrm{mg} / \mathrm{mL})$ was loaded in $\mathrm{NG}$ for in vivo experiments.

\subsection{Characterization techniques}

Functionalization of the polymers was evaluated by NMR and FT-IR analyses (Figs. S1, S2, S8). ${ }^{1} \mathrm{H}$ NMR spectra were run on a Bruker AC $(400 \mathrm{MHz})$ spectrometer, using deuterated chloroform $\left(\mathrm{CDCl}_{3}\right)$ or deuterium oxide $\left(\mathrm{D}_{2} \mathrm{O}\right)$, according to the solubility of the sample analyzed. Chemical shifts were reported as $\delta$ values in parts per million, with tetramethylsilane (TMS) as an internal reference. FT-IR spectra were recorded using a Thermo Nexus 6700 spectrometer coupled to a Thermo Nicolet Continuum microscope equipped with a $15 \times$ Reflachromat Cassegrain objective and the $\mathrm{KBr}$ pellet technique for the samples, at r.t. in air in the wave range $4000-500 \mathrm{~cm}^{-1}$, with 64 accumulated scans and resolution $4 \mathrm{~cm}^{-1}$.

Atomic force microscopy (AFM) was used to study NG (Fig. S9). The samples were prepared by dropping NG latexes onto silicon substrate, then drying. AFM images on $1 \times 1 \mu \mathrm{m}$ areas were recorded for the preliminary morphologic evaluation; $500 \times 500 \mathrm{~nm}$ images were cropped and a height line profile was drawn for each single gel. Surface morphology was evaluated by flattening the images (first order) using NTMDT software. NG sizes (volume-based), polydispersity index (PDI) and $\zeta$-potential were recorded using AFM and Dynamic Light Scattering (DLS). DLS analyses were performed with a Zetasizer Nano ZS from Malvern Instruments (Fig. S10): NG were dissolved in phosphate buffer saline solution $(0.01 \mathrm{M}, \mathrm{pH}=7.4)$ at $2 \mathrm{mg} / \mathrm{mL}$ then equilibrated for $60 \mathrm{~s}$ before data analysis at $37{ }^{\circ} \mathrm{C}$. Data provided the average of three measurements for each NG.

\subsection{Primary cell cultures}

Astrocyte-enriched primary cultures and neuron/astrocyte cocultures were obtained from spinal cord of 13-day-old C57BL/6 J mouse embryos (Charles River Laboratories International, Inc.) [17]. Briefly, the ventral horns of the embryonic spinal cord were isolated and treated with DNAse and trypsin (Sigma-Aldrich). After mechanical trituration and centrifugation with a cushion of bovine serum albumin (BSA), a heterogeneous population of neurons/glia was obtained. Another centrifugation step ( $800 \mathrm{xg}$ for $15 \mathrm{~min}$ ) performed through a $6 \%$ iodixanol pillow (OptiPrepTM; Sigma-Aldrich) was used to obtain a glial pellet and a narrow band at the top of the iodixanol pillow, corresponding to the fraction enriched with motoneurons. The glial pellet was plated at a density of 25,000 cells $/ \mathrm{cm}^{2}$ in $75 \mathrm{~cm}^{2}$ flasks previously coated with poly L-lysine $(10 \mu \mathrm{g} / \mathrm{mL}$, Sigma-Aldrich). Astrocyteenriched cultures were obtained by treating the glial cultures from which microglia cells had been eliminated by overnight (o.n.) shaking at $275 \mathrm{rpm}$, with $60 \mathrm{mM}$ L-leucine methyl ester (Sigma-Aldrich) for $90 \mathrm{~min}$. To prepare cultures to test the NG or a feeder layer for motor neuron cocultures, astrocytes were collected and seeded at a density of 50,000 cells $/ \mathrm{cm}^{2}$ in 24-well plates.

To establish motor neuron/astrocyte co-cultures, the motor neuronenriched fraction was seeded at a density of $10,000 \mathrm{cells} / \mathrm{cm}^{2}$ onto a mature astrocyte feeder layer [29].

Peritoneal macrophage cultures were obtained from 6 to 8-week-old C57 BL/6 J mice (Jackson Laboratory). Briefly, mice were euthanized by cervical dislocation, and peritoneal macrophages were harvested by rinsing the peritoneal cavity with $10 \mathrm{~mL}$ PBS (Life Technologies). After centrifugation at $400 \mathrm{xg}$ for $10 \mathrm{~min}$ cells were collected and plated into poly L-lysine pre-coated 24 -well plates $\left(100,000 \mathrm{cell} / \mathrm{cm}^{2}-\right.$ Sigma Aldrich).

\section{6. iPS-derived astrocyte cultures}

Episomal human iPS were obtained from GibcoTM (Life Technologies, CA, US, Lot V2.0), and cultured in feeder-free conditions in a xenofree culture medium formulation (StemMACS ${ }^{\mathrm{TM}}$ iPS-Brew XF, Miltenyi Biotec S.r.1.).

Using a commercial culture medium and in accordance with the 
literature [30], neural stem cells (NSC) were obtained after passaging and disgregation of iPS colonies. Briefly, confluent human iPS were detached with Accutase (GibcoTM, Life Technologies, CA, US) and split into six-well plates in a 1:3 ratio in iPS medium. The day after, culture medium was changed with enriched GibcoTM PSC Neural Induction Medium (Life Technologies) and maintained for 5-7 days before passaging. Primitive NSCs (pNSCs) were obtained from late passage (30-35) iPS after dissociation with Accutase (Life Technologies) and filtration through a $100 \mu \mathrm{m}$ strainer, then plated on Geltrex-coated dishes at a density of $0.5-1 \times 10^{5}$ cells $/ \mathrm{cm}^{2}$. Neurobasal medium and Advanced DMEM/F12 (1:1), with 2\% neural induction supplement (Life Technologies), were used to obtain stable NSC, which were then expanded for several passages and finally differentiated into astrocytes; to this purpose after the tenth passage NSC these were plated onto Geltrex-coated 24-well plates at a density of $5 \times 10^{4}$ cells $/ \mathrm{cm}^{2}$ and conditioned with an astrocyte differentiation medium (DMEM supplement with $1 \% \mathrm{~N}_{2}$, Glutamax and fetal bovine serum (FBS); Life Technologies) for 30 days.

\subsection{Culture treatments}

Mouse astrocytes, macrophages and neuron/astrocyte co-cultures were treated with $1 \mu \mathrm{g} / \mathrm{mL}$ of LPS (from Escherichia coli 0111:B4; Sigma-Aldrich) for $18 \mathrm{~h}$, as previously reported [17].

Human astrocytes culture was exposed to LPS $(10 \mu \mathrm{g} / \mathrm{mL}-$ Merck Life Science srl) for $18 \mathrm{~h}$ in medium with $10 \%$ serum. NG was then added to the cultures. To investigate the internalization mechanisms of NG, we treated astrocytes with chlorpromazine hydrochloride (CPZ $40 \mu \mathrm{g} / \mathrm{mL}$ Sigma-Aldrich) $2 \mathrm{~h}$ before NG exposure. To assess the NG degradation by lysosomes astrocytes were marked with Lysosensor dye (1:20.000 dilution, Life Technologies, cat.no. L7535) $24 \mathrm{~h}$ after exposure.

Finally, to investigate the loading capacity of NG, we treated the cultures of murine astrocytes with a solution of 1:50 of propidium iodide (PI, dilution 1:50 in water for injectable preparations, Sigma Aldrich) loaded in NG $(2.5 \%)$.

At the end of the treatment, primary cultures of murine astrocytes and macrophages, as well as human astrocyte cultures were fixed with 4\% paraformaldehyde for $40 \mathrm{~min}$ and stained with Fluorescein (FITC $0.1 \mu \mathrm{g} / \mathrm{mL}$; Sigma-Aldrich) by incubation at r.t. for $30 \mathrm{~min}$. Neuron/ astrocyte co-cultures were fixed with $4 \%$ paraformaldehyde for $40 \mathrm{~min}$ and incubated for $1 \mathrm{~h}$ at r.t. in the blocking solution (PBS, $0.2 \%$ Triton X100 (Sigma-Aldrich) and 1\% FBS (Sigma-Aldrich)) followed by 1:1000 anti-SMI-32 primary antibody (mouse; BioLegend) in PBS at r.t. for $4 \mathrm{~h}$. The cells were then washed with PBS and the fluorescence was detected using a specific secondary antibody conjugated to fluorophores (goat anti-mouse; Alexa Fluor 594, 1:500, r.t. for 1 h; Invitrogen). Cell nuclei were labeled with $250 \mathrm{ng} / \mathrm{mL}$ Hoechst 33258 (Invitrogen).

\subsection{NG internalization}

To quantify the internalization of NG in cell cultures, images were randomly acquired using a Cell $\mathrm{R}$ microscope (Olympus) equipped with $60 \times$ magnification and an ORCA camera (Hamamatsu). The fluorescent signal was quantified using free Fiji software (http://fiji.sc/Downloads) or Imaris software (Bitplane). Fluorescein was used to measure the cytosol area of single cells (about 30-50 cells for each experiment) and Hoechst to stain cell nucleus. NG internalization was evaluated as the percentage of $\mathrm{RhB}$ or Cy5 area (NG signal area/total cell area) or NG signal expressed as $\mu \mathrm{m}^{2}$ /single cell after image segmentation.

In order to evaluate the NG internalization overtime, time lapse analysis was done (Olympus microscope IX81 equipped with $60 \times$ magnification, ORCA Camera Hamamatsu and Cell Sense software). The NG uptake was assessed with Imaris Software (Bitplane) and the data plotted and analyzed with Graphpad Prism. We also confirmed cell uptake analyzing free NG remained in the medium by microplate readers (Tecan Infinite $200 \mathrm{PRO}, \mathrm{RhB} \lambda_{\mathrm{Em}} 590$ or Cy $5 \lambda_{\mathrm{Em}}$ 680) expressed as NG percentage found in the medium after $24 \mathrm{~h}$ of NG exposure.

\subsection{Colocalization study}

Colocalization was studied in vitro on about 90 cells sampled in six frames $\left(145^{*} 110 \mu \mathrm{m}\right)$, and ex vivo, one day after NG injection an area of $13,220 \mu \mathrm{m}^{2}$ was examined in three sections, $30 \mu \mathrm{m}$ thick, sampled one every two sections. Colocalization was quantified by Pearson's coefficient (Imaris Software, Bitplane).

\subsection{Drug loading procedures}

To optimize drug loading two different strategies were employed. In the first, the drug was entrapped in the swollen preformed NG as follows: a Polytetrafluoroethylene (PTFE) cylinder $1 \mathrm{~cm}$ in diameter and 1 $\mathrm{cm}$ long with an axial perforation of $1 \mathrm{~mm}$ diameter and a radial one of $500 \mu \mathrm{m}$ was used for mixing (Fig. S8, a). The NG water suspension (1 $\mathrm{mg} / \mathrm{mL})$ and the dissolved Rolipram drug solution in ethanol $(0.014 \mathrm{mg} /$ $\mathrm{mL}$ ) were loaded in syringe pumps and injected radially into the device at a flow rate of $1 \mathrm{~mL} / \mathrm{min}$. For the second procedure lyophilized NG was suspended directly in drug solution and mixed until dissolution. The swelling NG establishes high concentration gradient between the solution and the inner core of the NG (Fig. S8, b). Then, loaded NG was dialyzed (membrane $\mathrm{Mw}$ cutoff $=3500 \mathrm{Da}$ ) against aqueous solution for $30 \mathrm{~min}$ in order to remove free drug molecules. Rolipram content was checked by high-performance liquid chromatography (HPLC; Waters, Milford, MA) in dimethylsulfoxide (DMSO). Loading efficiency (\% loading) was calculated with the equation:

\%loading $=\frac{\text { drug entrapped within } N G}{\text { initial drug loaded }} \cdot 100$

For drug delivery study, each nanogel sample within dialysis bag, was placed in excess of PBS and aliquots $(3 \times 100 \mu \mathrm{l})$ were collected at defined time points, while the sample volume was replaced by fresh solution, in order to avoid mass-transfer equilibrium with the surrounding release environment. The experiments were performed at $37{ }^{\circ} \mathrm{C}$. Percentages of released Rolipram were then measured by HPLC.

\subsection{Animal care}

All procedures involving care and use of laboratory animals were conducted in conformity with Italian Governing Law (D.lgs 26/2014; Authorization n.19/2008-A issued March 6, 2008 by the Ministry of Health); the NIH Guide for the Care and Use of laboratory Animals (2011 edition) and EU directives and guidelines (EEC Council Directive 2010/ 63/UE). In addition, the Istituto di Ricerche Farmacologiche Mario Negri IRCCS provides internal authorization for persons conducting animal experiments (Quality Management Certificate - UNI EN ISO 9001:2015 - Reg. No.6121).

\subsection{Surgery}

B6.129P-Cx3cr1tm1Litt/J (Charles River Laboratories International, Inc.) mice were used for in vivo experiment. The animals received a subcutaneous injection of antibiotic (50 mg/ $\mathrm{kg}$ of ampicillin) and analgesic $(0.15 \mathrm{mg} / \mathrm{kg}$ of buprenorphine). All surgical procedures were carried out in deep anesthesia intraperitoneal injection of hydrochloride (IMALGENE, $100 \mathrm{mg} / \mathrm{kg}$ ) and medetomidine hydrochloride (DOMITOR, $1 \mathrm{mg} / \mathrm{kg}$ ).

Briefly, mice were placed on a Cunningham Spinal Cord Adaptor (Stoelting, Dublin, Ireland), mounted on a stereotaxic frame, and the lumbar spinal cord was exposed by laminectomy at the T12 vertebra. Trauma was induced using an aneurysm clip (2 Biological Instruments snc, cat. N. 14,120) left in place for $1 \mathrm{~min}$, applying a force of $30 \mathrm{~g}$. After one day, NG free or loaded with Rolipram was injected intraparenchymally (six $0.250 \mu \mathrm{L}$ injections) with a glass capillary (outer 
diameter $40 \pm 2 \mu \mathrm{m}$ ) to cover the injured area.

Finally, after both treatments (clip or injections), dorsal muscles were juxtaposed using absorbable sutures and the skin was sutured and disinfected. Not injured mice were used for assessment of NG internalization and four injections were done, exposing the spinal cord after laminectomy. After surgery, the animals were placed in separate cages for recovery.

\subsection{Spinal cord transcardial perfusion}

For assessments of astrocytosis in spinal cord the mice were euthanized at fourteen days post-injury (dpi); the study of internalization in vivo was done in mice perfused one day after the NG injection. Briefly, under deep anesthesia (IMALGENE, $100 \mathrm{mg} / \mathrm{kg}$ and DOMITOR, $1 \mathrm{mg}$ / $\mathrm{kg}$ ) mice were transcardially perfused for 4 min with $40 \mathrm{~mL}$ of PBS 0.1 $\mathrm{mol} / \mathrm{L}, \mathrm{pH} 7.4$ and $50 \mathrm{~mL}$ of paraformaldehyde solution (4\%) in PBS for $5 \mathrm{~min}$. Spinal cord was extracted and post-fixed o.n. in the same fixative at $4{ }^{\circ} \mathrm{C}$. One day later, the tissue was transferred to $30 \%$ sucrose in 0.1 mol/L PBS o.n. and stored at $4{ }^{\circ} \mathrm{C}$ until use.

\subsection{Immunofluorescence}

The spinal cord was embedded for histological analysis, in OCT compound, frozen in $\mathrm{N}$-pentane at $-45{ }^{\circ} \mathrm{C}$ for $3 \mathrm{~min}$, and stored at $-80^{\circ} \mathrm{C}$ until use. To evaluated astrogliosis, frozen tissue was sectioned at $30 \mu \mathrm{m}$ using a cryostat at a temperature of $-20^{\circ} \mathrm{C}$, starting from about $6 \mathrm{~mm}$ rostral to the epicenter, and sections were collected in PBS. Twenty serial sections were chosen, one for each five, and separated for immunofluorescence. For colocalization studies tissue was sectioned at $60 \mu \mathrm{m}$; then only positive slices at the Cy5 signal were collected separately and GFAP staining was done.

Sections were incubated with primary antibody directed against astrocytes (Glial Fibrillary Acidic Protein (GFAP); 1:1000 dilution, Millipore) or neurons (NeuN, 1:1000 dilution, Merck Life Science S.r.l.) dissolved in PBS, $1 \%$ normal goat serum (NGS; Sigma Aldrich) and $0.1 \%$ Triton X-100, and incubated o.n. at $4{ }^{\circ} \mathrm{C}$. The next day, the sections were washed in PBS and the fluorescence was detected using a secondary fluorescence antibody (Alexa Fluor 594 or 488, 1:500; Invitrogen). Spinal cord slices were cover slipped with FluorSave Reagent (Millipore) before acquisition at $40 \times$ or $60 \times$ magnification by confocal microscopy (Olympus Fv1000, Laser 594 or 488).

\subsection{Astrocytosis}

The 20 sections at $14 \mathrm{dpi}$, stained with GFAP, were acquired using a Cell $\mathrm{R}$ microscope (Olympus). Acquisitions were done with $40 \times$ magnification using a 3D mosaic imaging technique. On each section a frame was superimposed sampling a grid $(200 * 200 \mu \mathrm{m})$ containing astrocytes marked with GFAP. To quantify GFAP staining in each frame we used an unbiased counting probe $(216 * 165 \mu \mathrm{m})$ and the Cavalieri method (Imagej software). Astrocyte volume was calculated as: $\mathrm{V}=\Sigma$ $\mathrm{P} * \mathrm{~A} * \mathrm{~T}(\mathrm{~V}$, volume; $\mathrm{P}$, number of points hitting GFAP positive signal; A, distance between the grid points and $\mathrm{T}$, distance between each section sampled). P was calculated as the average of five replicates; A was 400 $\mu \mathrm{m}$ (the slice interval was $6 \mu \mathrm{m}$ ) and $\mathrm{T}$ was $50 \mu \mathrm{m}$. Finally, the Coefficient of Error (CE) and the Coefficient of Variation (CV) [31,32] were reported as mean CE: no treated injury 0.067; Rolipram loaded NG 0.085; CV: no treated injury 0.265 , Rolipram loaded NG 0.285.

\subsection{Statistical analyses}

For statistical analyses, we used Prism software (Graphpad). The Mann-Whitney test, Student's $t$-test and Kruskal-Wallis test followed by Dunn's multiple comparisons test were used.

\section{Results}

\section{1. $N G$ and the targeting moieties}

NG were synthesized by means of the $\mathrm{CH}_{2} \mathrm{Cl}_{2}$-in-water emulsification-evaporation method. Briefly, activated PEG-CDI was dissolved in the organic phase, and PEI functionalized with propargyl moieties reacted via 1,3-Huisgen cycloaddition with RhB or Cy5 azides, then dipped in aqueous solution. After sonication, with the progressive evaporation of $\mathrm{CH}_{2} \mathrm{Cl}_{2}$ in the emulsion status, the system ensures homogeneous dispersion of PEG chains around PEI, and promotes the interactions between the imidazole and amine moieties of the two polymers, giving rise to the formation of carbamate bonds and entanglement of the chains, forming a colloidal dispersion hereafter named NG-RhB and NG-Cy5. In a further synthetic step, NG were coated with a linker bearing terminal primary amines, that was grafted in a single step to either NG-RhB or NG-Cy5 dissolved in water by direct addition of 3bromopropylamine, stirring at room temperature for $17 \mathrm{~h}$ the final solutions. These colloidal dispersions were named $\mathrm{NH}_{2}+\mathrm{RhB} \mathrm{NG}$ and $\mathrm{NH}_{2}+\mathrm{Cy} 5 \mathrm{NG}$. The 3-bromopropylamine nucleophilic substitution occurred on the residual PEI secondary amine groups and this reaction did not affect the NG bonds, preserving the structural organization of the polymeric chains, as already discussed in our previous works [24,27]. The reaction scheme for the NG synthesis is reported in Fig. S7. The NG physical features were investigated using dynamic light scattering (DLS) as visible from the Table S1 (Supplementary data). The distributed positive charges in $\mathrm{NH}_{2}$-coated $\mathrm{NG}$ were related to the presence of $-\mathrm{NH}_{2}$ surface groups that gave rise to NG protonation and a positively charged interface.

\subsection{Cellular uptake of targeting moieties in murine astrocytes, macrophages and neurons in vitro}

Murine astrocytes, macrophages and neurons were activated with LPS for $18 \mathrm{~h}$ in order to analyze these cells in an inflammatory state. The uptake of NG functionalized with different moieties $\left(\mathrm{NH}_{2}, \mathrm{RhB}\right.$ or $\left.\mathrm{Cy} 5\right)$ was examined by a time-lapse analysis in order to find the maximum NG internalization in the cytosol. Primary cultures of astrocytes or macrophages isolated from the spinal cord of mouse embryos were recorded up to $24 \mathrm{~h}$ after the NG exposure (Fig. S12). NPs internalization was measured at $24 \mathrm{~h}$, when fluorescent signal reached the maximum for each NG tested (Fig. S12). NG internalization and distribution was found mostly in the cytosol of the cell examined, as confirmed by 3D cell reconstruction after $24 \mathrm{~h}$ of exposure (Fig. S13). The percentage of NG signal was measured as fluorescent signal area over the total cell area. A large amount of $\mathrm{RhB}$ or $\mathrm{NH}_{2}+\mathrm{RhB}$ functionalized $\mathrm{NG}$ was found in the cytosol of astrocytes (Fig. 1a-h,q,r). Uptake was significantly lower when astrocytes were exposed to $\mathrm{Cy} 5$ or $\mathrm{NH}_{2}+\mathrm{Cy} 5$ (Fig. 1i-r) compared to $\mathrm{RhB}$. In order to confirm a reduced uptake when Cy5 was conjugated with NG we treated astrocytes with $\mathrm{NH}_{2}+\mathrm{RhB} \mathrm{NG}$ and $\mathrm{NH}_{2}+\mathrm{Cy} 5 \mathrm{NG}$ and recorded the NG internalization by time-lapse analysis. At the end of the analysis $(24 \mathrm{~h})$ a significant lower amount of $\mathrm{NH}_{2}+$ Cy5 NG is detected in the cytosol of astrocytes compared to $\mathrm{NH}_{2}+\mathrm{RhB}$ NG (Fig. S14; A, B, C). $\mathrm{NH}_{2}+\mathrm{Cy} 5 \mathrm{NG}$ internalization was measured also in astrocytes not treated with LPS. The quantification showed a reduced quantity of NG internalized, significantly lower when compared to cells activated with LPS (Fig. S15).

When we tested NG on macrophages, a certain amount of NG-RhB and $\mathrm{NH}_{2}+\mathrm{RhB}$ NG was internalized in the cytosol (Fig. 2a-h,q,r) but was significantly limited when we conjugated $\mathrm{Cy} 5$ or $\mathrm{NH}_{2}+\mathrm{Cy} 5$ to NG (Fig. 2i-r), whereas no significant difference was found for $\mathrm{NH}_{2}+\mathrm{RhB}$ NG $v s$ NG-RhB.

To confirm a reduced uptake for NG conjugated Cy5 we also treated macrophages with $\mathrm{NH}_{2}+\mathrm{RhB} \mathrm{NG}$ and $\mathrm{NH}_{2}+\mathrm{Cy} 5 \mathrm{NG}$. Twenty-four hours after the exposure a significant lower amount of $\mathrm{NH}_{2}+\mathrm{Cy} 5 \mathrm{NG}$ is detected in the cytosol of macrophages compared to $\mathrm{NH}_{2}+\mathrm{RhB} \mathrm{NG}$ 


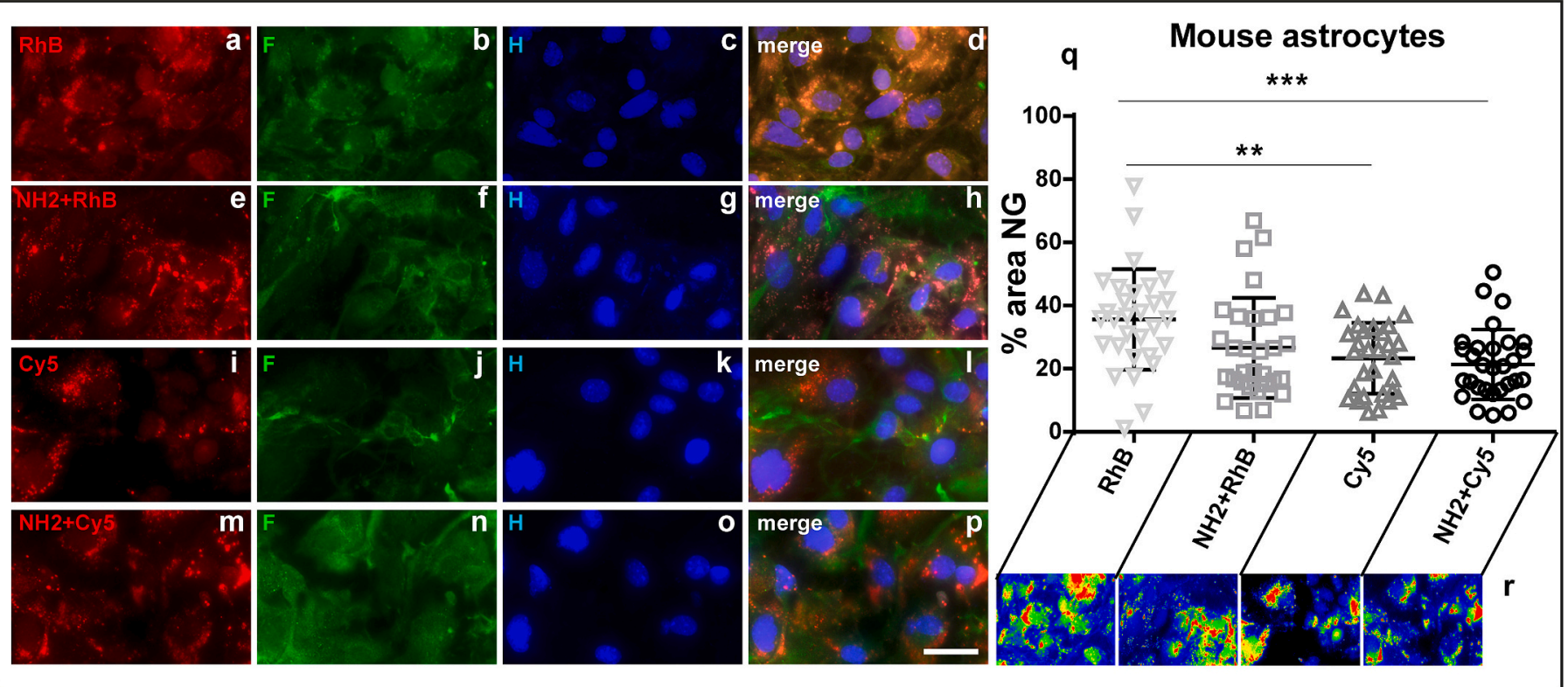

Fig. 1. Contributions of different moieties to NG uptake in murine astrocytes, in vitro.

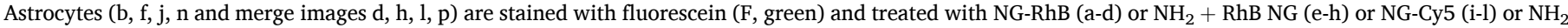

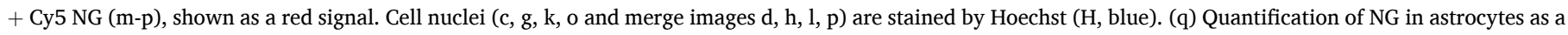

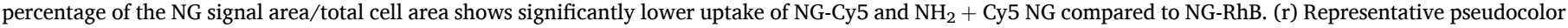

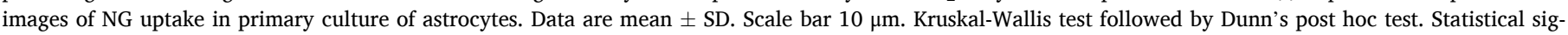

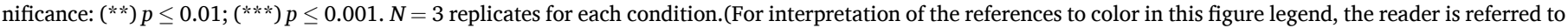
the web version of this article.)

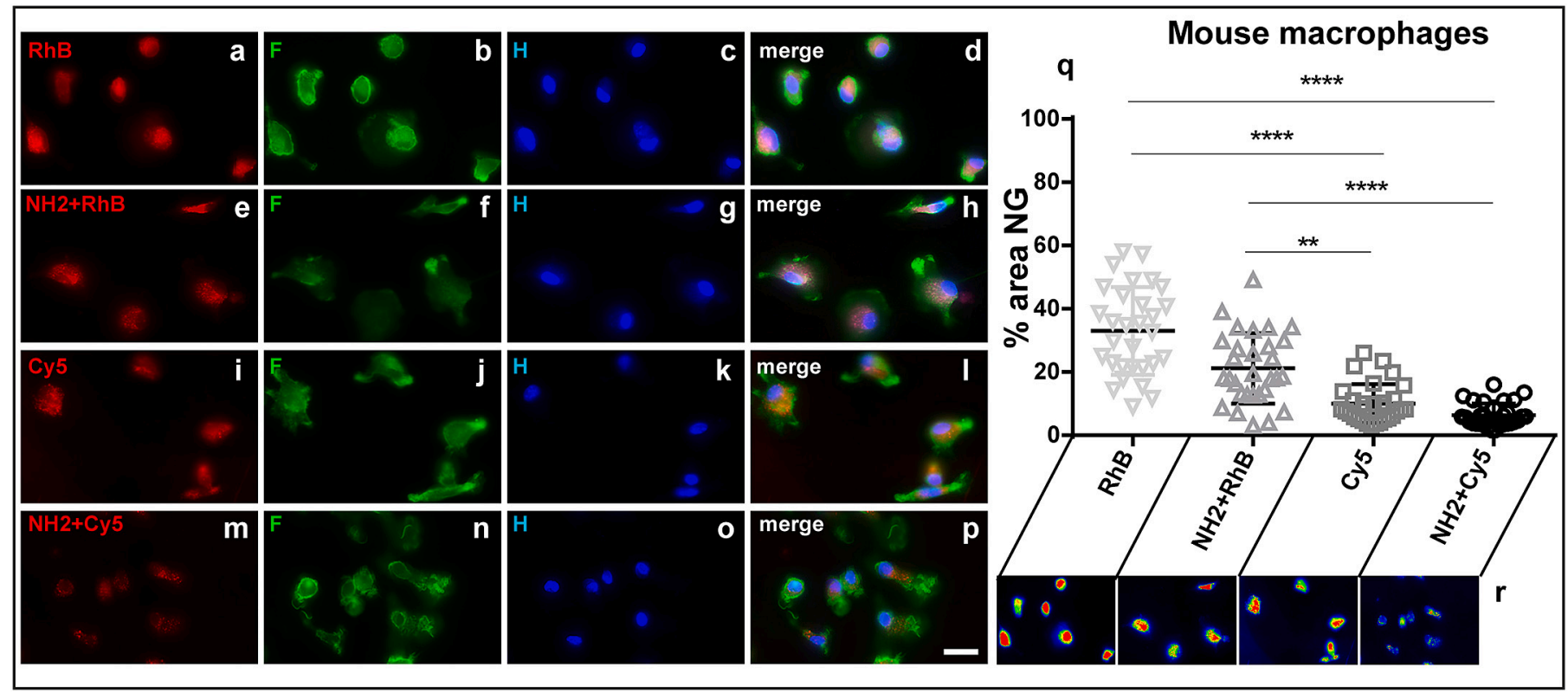

Fig. 2. Contributions of different moieties to NG uptake in murine macrophages in vitro.

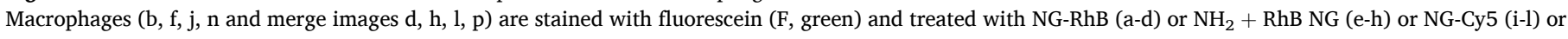

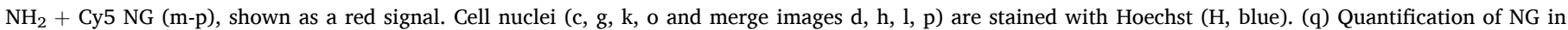

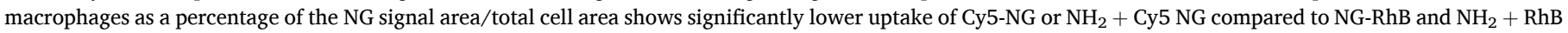

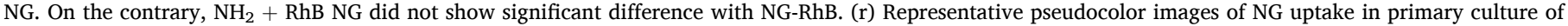

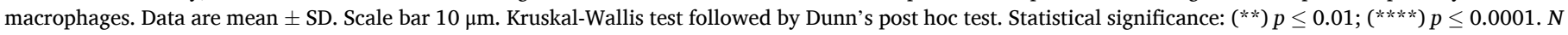
$=3$ replicates for each condition.(For interpretation of the references to color in this figure legend, the reader is referred to the web version of this article.)

(Fig. S14; D-F).

Comparing the uptake of astrocytes and macrophages expressed as $\mu \mathrm{m}^{2}$ of the cell signal (Fig. 3A, B), the $\mathrm{NH}_{2}+\mathrm{Cy} 5 \mathrm{NG}$ formulation besides suppressing macrophage uptake remained more internalized in astrocytes as shown by the 3.2 ratio of NG internalized for astrocytes/ macrophages (Fig. 3C, d). NG formulated with RhB gave a ratio of 2.7 (Fig. 3C, a), $\mathrm{NH}_{2}+\mathrm{RhB}$ a ratio of 2.4 (Fig. 3C, b) and $\mathrm{Cy} 5$ a ratio of 2.7 (Fig. 3C, c). This conclusion was further supported by observing the 


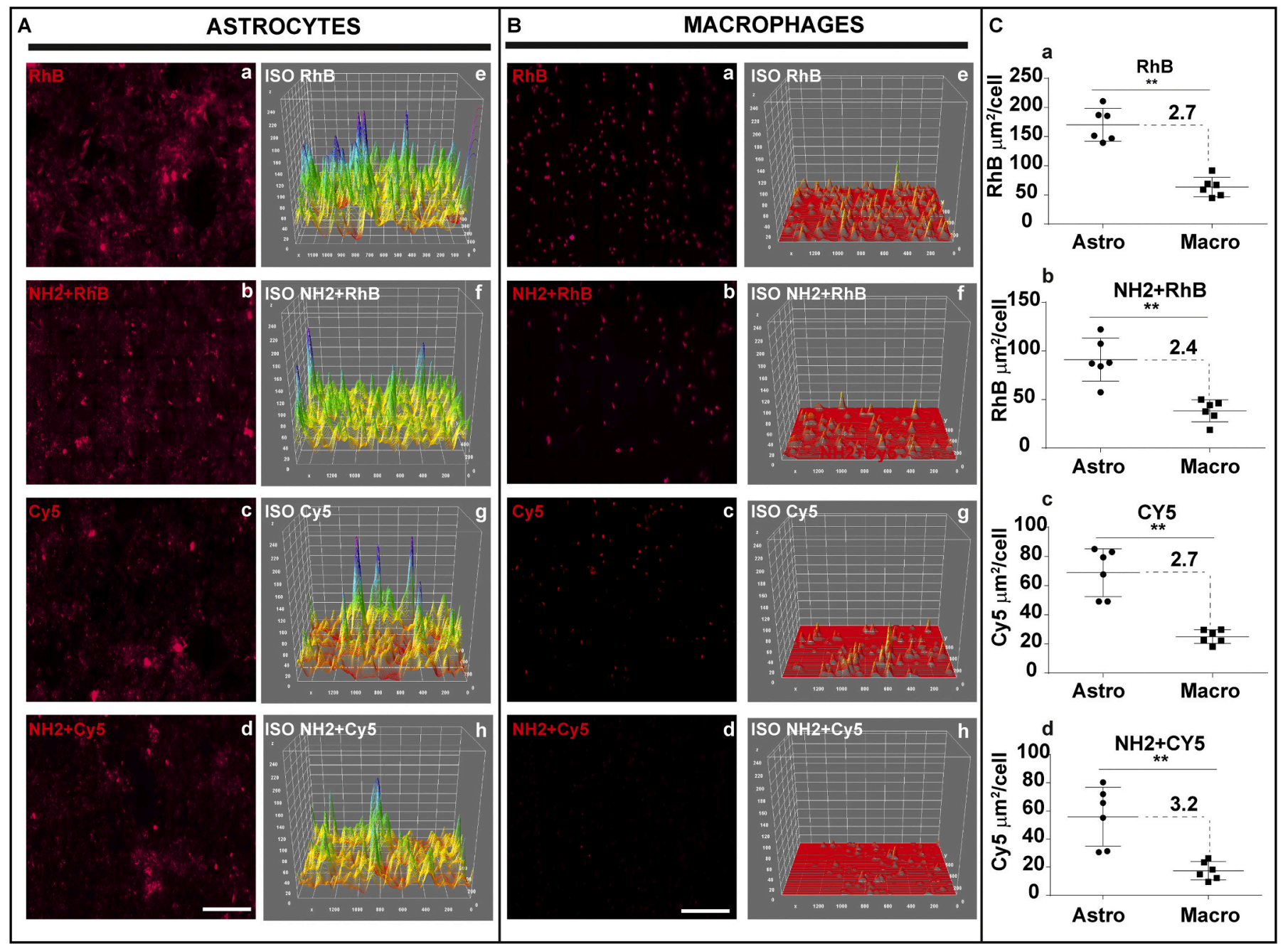

Fig. 3. Comparative study of the contributions of different moieties to NG uptake in murine astrocytes and macrophages in vitro.

$\mathrm{RhB}$ (a), $\mathrm{NH}_{2}+\mathrm{RhB}$ (b), $\mathrm{Cy} 5$ (c), $\mathrm{NH}_{2}+\mathrm{Cy} 5$ (d) $\mathrm{NG}$ are shown as a red signal internalized in astrocytes (A) or macrophages (B) and their relative isosurface representations (e-h). (C) Quantification of $\mathrm{RhB}$ (a), $\mathrm{NH}_{2}+\mathrm{RhB}$ (b), $\mathrm{Cy} 5$ (c), $\mathrm{NH}_{2}+\mathrm{Cy} 5$ (d) $\mathrm{NG}$ uptake in astrocytes and macrophages expressed as the ratio of the average areas of the fluorescent signal in astrocytes to macrophages. $\mathrm{NH}_{2}+\mathrm{Cy} 5 \mathrm{NG}$ had a higher astrocyte/macrophage internalization ratio than to $\mathrm{RhB}, \mathrm{NH} \mathrm{H}_{2}+\mathrm{RhB}$ and Cy5. Data are mean \pm SD. Mann-Whitney test. Scale bar $30 \mu \mathrm{m}$. Statistical significance: $(* *) p \leq 0.01 . N=3$ replicates for each condition.

isosurfaces in Fig. 3(A, B). NG fluorescent signals were mapped onto a threedimensional intensity plot to better show the quantity of NG signal internalized. The amount of internalized signal intensity is shown by pseudo color from red (low signal intensity) to blue (high signal intensity) (Fig. 3A, e-h; B, e-h). In order to confirm the different NG uptake in astrocytes or macrophages, we quantified the NG fluorescent signals left in the medium after the NG cell uptake within $24 \mathrm{~h}$ demonstrating again that $\mathrm{NH}_{2}$ and $\mathrm{Cy} 5$ could reduce NG internalization in astrocytes and macrophages (Fig. S9; B, D).

We also exposed neurons in vitro to different NG formulations, but we did not detect any signal inside the cells (Fig. S13).

3.3. Cellular uptake of targeting moieties in induced pluripotent stem cells (iPS)-derived human astrocytes in vitro

To verify whether our designed nanocarriers were appropriate for human cells, we treated human (iPS)-derived astrocytes with LPS for 18 $\mathrm{h}$ and then with NG functionalized with different ligands $\left(\mathrm{NH}_{2}, \mathrm{RhB}\right.$ or Cy5), as previously examined on murine cells. Human astrocytes were examined after $24 \mathrm{~h}$ of exposure to NG. NG internalization was evaluated as the percentage of NG signal area over the total cell area. NG internalization and distribution was mostly in the cytosol as for murine astrocytes. We found a larger amount of RhB conjugated NG in the cytosol of astrocytes (Fig. 4a-d, q, r) than $\mathrm{NH}_{2}+\mathrm{RhB}$ (Fig. 4e-h, q, r) or $\mathrm{NH}_{2}+\mathrm{Cy} 5$ (Fig. $4 \mathrm{~m}-\mathrm{p}, \mathrm{q}, \mathrm{r}$ ) specimens confirming that the $\mathrm{NH}_{2}$ functional group partially limited nanovector uptake in astrocytes. Unlike in the murine model, the Cy5 moiety did not affect the internalization compared to RhB (Fig. 4a-d, i-l, q, r).

\subsection{Internalization, degradation and delivery of Cy5 conjugated to NG in murine astrocytes in vitro}

As previously demonstrated, $\mathrm{NH}_{2}$-Cy5 coated $\mathrm{NG}$ was the most promising for targeting astrocytes, reducing microglia/macrophage recognition and uptake. So we decided to characterize internalization and degradation only of this moiety. LDH assay was also be used to demonstrate that $\mathrm{NH}_{2}+\mathrm{Cy} 5$ coated NG were not toxic to astrocytes (Fig. S17). Furthermore, we investigated how $\mathrm{NH}_{2}+\mathrm{Cy} 5$ coated NG were to be internalized by the cells in murine astrocytes in vitro. We examined Cy5 far-red signal after pretreatment with a clathrin-mediated pinocytosis inhibitor (chlorpromazine, CPZ, that causes the assembly of adaptor proteins and clathrin on membranes, blocking endocytosis) and subsequent exposure to $\mathrm{NG}$ for $24 \mathrm{~h}$. $\mathrm{NH}_{2}+\mathrm{Cy} 5 \mathrm{NG}$ in the cytosol were significantly lower in astrocytes treated with CPZ (Fig. 5A; e-i) then in 

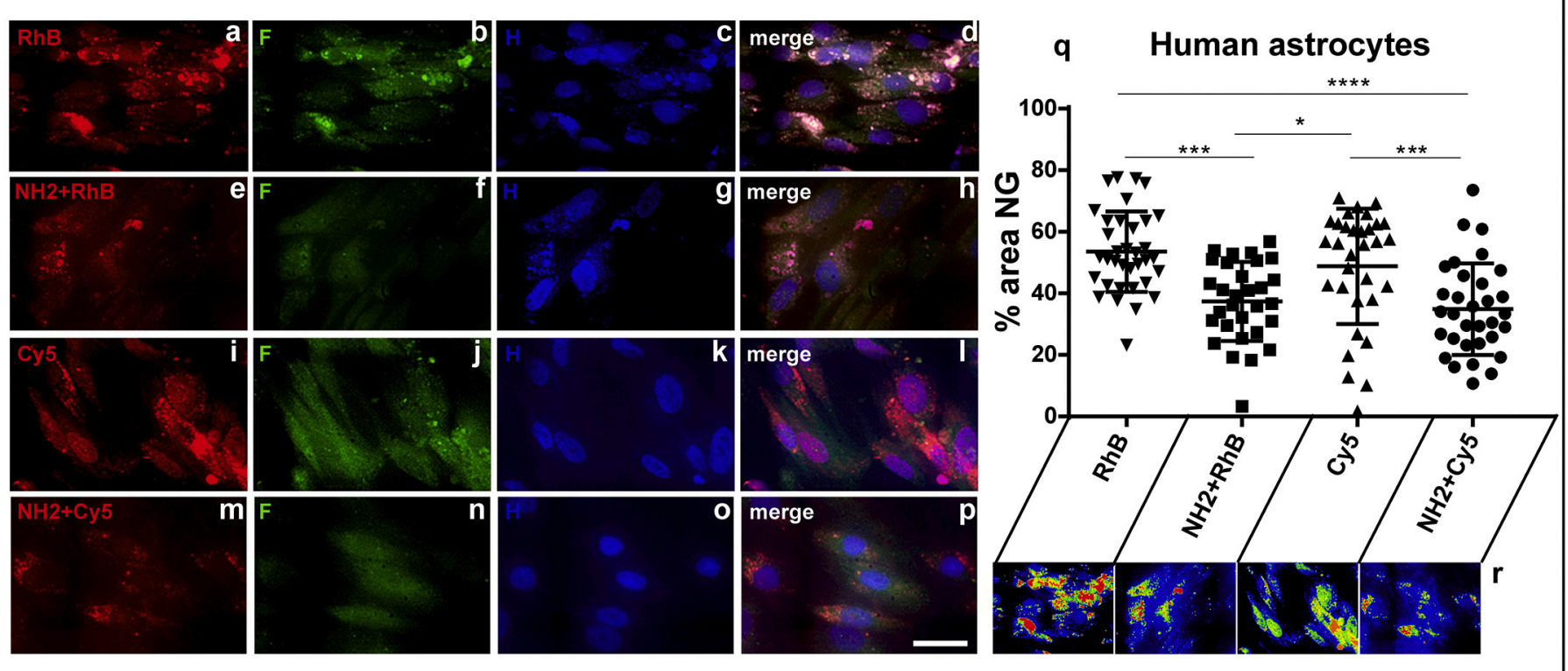

Fig. 4. Contribution of different moieties to NG uptake in human astrocytes in vitro.

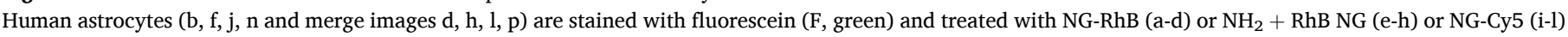

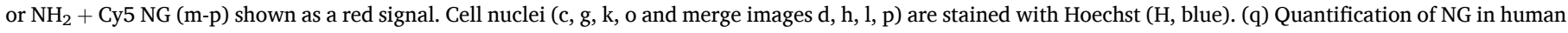

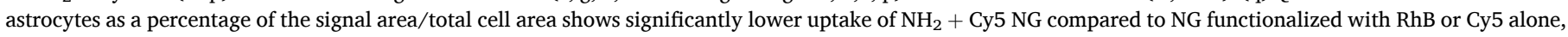
but not with $\mathrm{NH}_{2}+\mathrm{RhB}$ specimen.

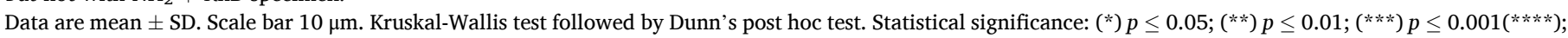

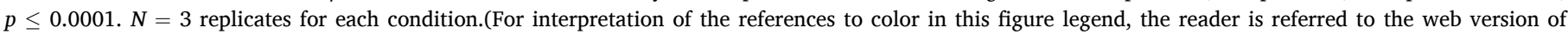
this article.)

untreated cells (Fig. 5Aa-d, i), suggesting a mechanism of clathrinmediated endocytosis in NG uptake.

Lysosomes form the primary compartment that degrades extracellular material after clathrin-mediated endocytosis. We investigated the lysosomal machinery in murine astrocytes after exposure to $\mathrm{NH}_{2}+\mathrm{Cy} 5$ conjugated NG. A lysosensor was used to record the enzymatic activity. After $24 \mathrm{~h}$ there was a pronounced increase in lysosomal activity (Lyso, green) (Fig. 5Bb). Examining the $\mathrm{NH}_{2}+\mathrm{Cy} 5$ conjugated NG distribution we found marked colocalization between the lysosensor (green signal) and NG (red signal) (Fig. 5Ba-c), confirmed by Pearson's correlation coefficient (Fig. 5Bd). These data demonstrated that a pathway consisting of clathrin-mediated endocytosis and degradation by lysosomal enzymatic activity is involved for $\mathrm{NH}_{2}+\mathrm{Cy} 5$ conjugated NG when internalized into astrocytes.

$\mathrm{NH}_{2}+$ Cy5 coated NG were also evaluated for their ability to deliver molecules. NG were loaded with propidium iodide (PI), which is impermeable and unable to cross the membrane of live cells. We exposed astrocytes to PI-loaded NG for $24 \mathrm{~h}$. There was a localized NG signal in the cytosol of astrocytes, and a more diffuse PI signal in the same cells suggesting a passage from the outside to the inside mediated by NG (Fig. 5C, a-f). Pearson's coefficient was used to demonstrate the overlap between them (Fig. 5C, g). We found partial colocalization of the two signals suggesting that PI once inside the cells was delivered and diffused in the cytosol.

\subsection{Cellular distribution and uptake of NG with targeting moieties in vivo}

To investigate the distribution of $\mathrm{RhB}, \mathrm{NH}_{2}+\mathrm{RhB}$, Cy5 and $\mathrm{NH}_{2}+$ Cy5 coated NG in vivo, NG was given $24 \mathrm{~h}$ after the trauma by six injections equally distributed at a distance of $-1.5 /+1.35 \mathrm{~mm}$ from the epicenter of the injured site. We investigated NG distribution and internalization three days after the injection. The RhB or Cy5 signal was used to follow NG distribution ex vivo in sections of damaged spinal cord (Fig. 6B). Structural markers were used to identify astrocytes (GFAP)
(Fig. 6A) and neuronal cells (neurotrace, Neu) (Fig. S15); microglia/ macrophages were distinguished as GFP-positive cells (Cx3CR1 transgenic mice) (Figs. 6A, S15). Activated astrocytes were detected mostly in the white matter, whereas activated microglial cells were distributed overall in the sections of the damaged spinal cord. In astrocytes a colocalized signal was detected between GFAP and RhB conjugated with NG (Fig. 6A, a,b,d) or $\mathrm{NH}_{2}+\mathrm{RhB}$ conjugated with NG (Fig. 6A, e,f,h) or Cy5 conjugated with NG (Fig. $6 \mathrm{~A}, \mathrm{i}, \mathrm{j}, \mathrm{l})$ or $\mathrm{NH}_{2}+\mathrm{Cy} 5$ conjugated with NG (Fig. 6A, m,n,p), but not with neurons (Fig. S15; d,h,l,p), as also demonstrated by Pearson's coefficient which was close to zero, in line with previous in vitro findings (Fig. S16; b). A few neurons in the epicenter of the lesion had NG in the cytosol (data not shown), quite likely due to the impaired integrity of the membrane of these cells in the most damaged part of the spinal cord. In contrast, microglia/macrophages were also involved in the uptake of some NG in vivo. $\mathrm{RhB}, \mathrm{NH}_{2}+$ RhB or Cy5 conjugated NG showed partial colocalization with the GFP signal, whereas $\mathrm{NH}_{2}+\mathrm{Cy} 5$ significantly reduced NG uptake in microglia/macrophages compared to RhB. This was confirmed by Person's coefficient, which was significantly different from RhB conjugated to NG and close to zero for $\mathrm{NH}_{2}+\mathrm{Cy} 5$ conjugated to NG (Fig. S16; a).

\subsection{Efficacy of rolipram loaded in Cy5 functionalized ng in vivo}

To validate the ability of the nanovector system to act pharmacologically on astrocytes in vivo, we loaded $\mathrm{NH}_{2}+\mathrm{Cy} 5 \mathrm{NG}$ with Rolipram, an anti-inflammatory drug that prevents NF-kB activity and proinflammatory cytokine release [25]. Rolipram was loaded with a procedure to maximize the internalization of the drug into the NG, limiting its free dispersion in the suspension (Fig. S4). The percentage of Rolipram loaded in NG was $72.8 \pm 3.5 \%$ with the first procedure and $80.5 \pm$ $2.5 \%$ with the second (see Experimental sections, drug loading procedures). Representative release profile is visible in Fig. S22 and no differences are detectable between the different conditions (presence of coating and loading procedure). Astrocytes were evaluated counting 


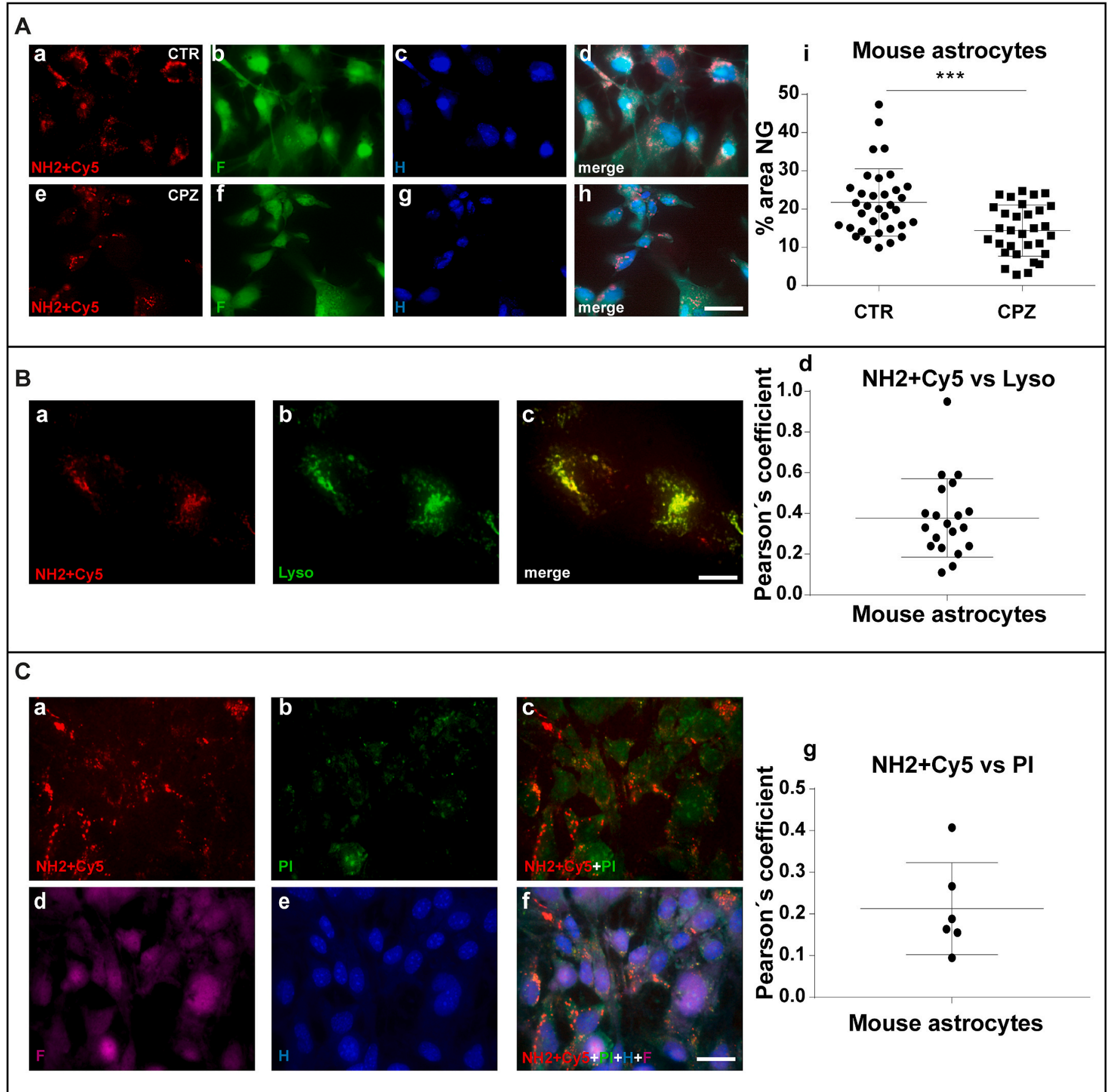

Fig. 5. Internalization, degradation and compound delivery of $\mathrm{NH}_{2}+\mathrm{Cy} 5$ conjugated to NG in murine astrocytes in vitro.

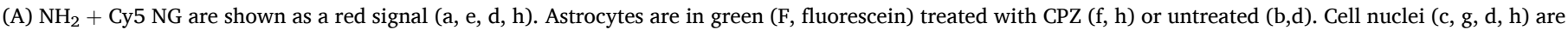

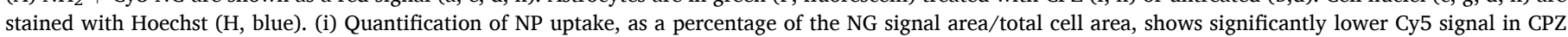

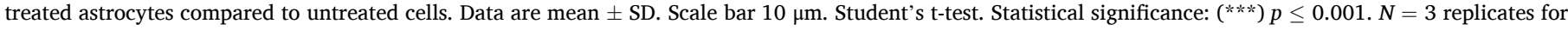
each condition.

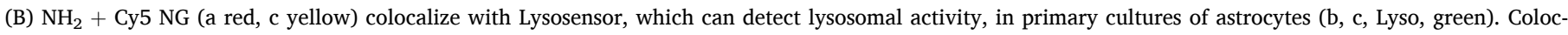
alization was confirmed by quantitative analysis using Pearson's coefficient (d). Scale bar $5 \mu \mathrm{m}$. $N=3$ replicates for each condition.

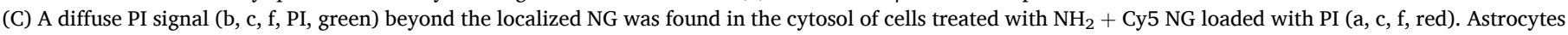

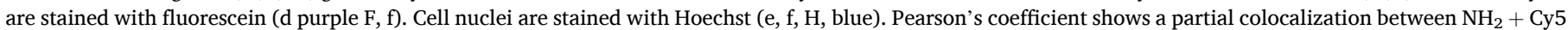

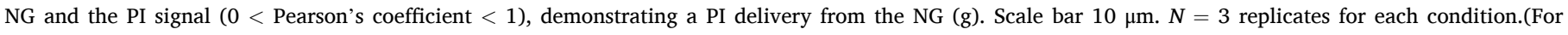
interpretation of the references to color in this figure legend, the reader is referred to the web version of this article.)

GFAP-positive cells by quantitative stereological analysis (see Experimental sections) around the epicenter of the injury for a rostro-caudal distance of $-1.50 /+1.35 \mathrm{~mm}$ (Fig. $7 \mathrm{~g}$ ). We compared astrocytosis of the ex-vivo spinal cord from untreated injured mice (INJ) (Fig. 7a-c, g, h) with mice given Rolipram loaded in $\mathrm{NH}_{2} \mathrm{Cy} 5$ conjugated to NG (Rolipram_NG) (Fig. 7d-h) 14 days post-injury (DPI). In the GFAP staining profile INJ mice had more astrocytosis than to Rolipram_NG in the whole rostro-caudal tract (Fig. $7 \mathrm{~g}$ ). This was confirmed by the 

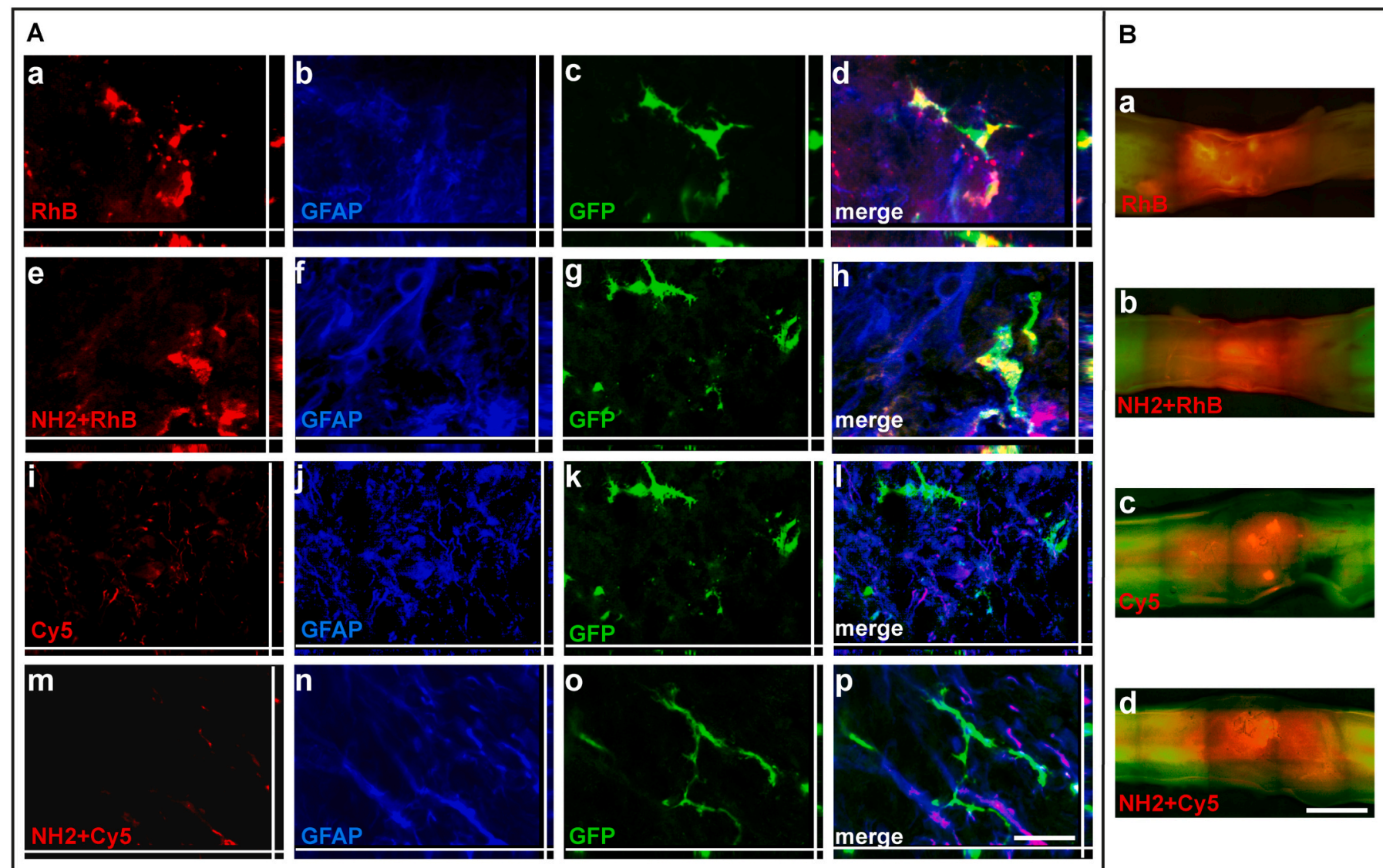

Fig. 6. Contributions of different moieties to NG uptake in astrocytes or microglia in vivo.

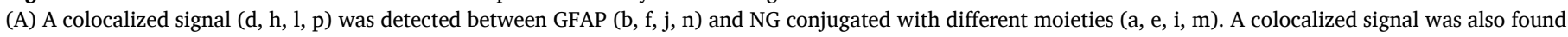

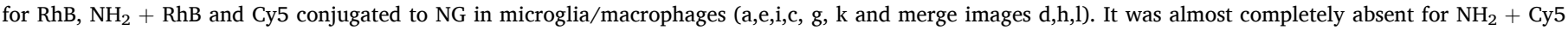

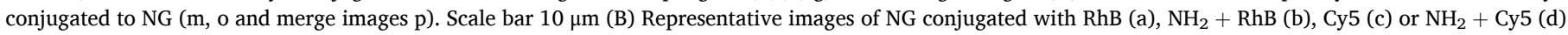
distributed in a spinal cord one day after injection (side view). Scale bar $1500 \mu \mathrm{m} . N=5$ for each group.

significant difference in the overall quantitative GFAP staining for the two groups (Fig. 7h). This suggests astrocyte activation is substantially reduced after treatment with Rolipram delivered by $\mathrm{NH}_{2}$-Cy5 conjugated to NG, confirming the nanovector's ability to deliver therapeutic molecules in astrocytes.

\section{Discussion}

Various studies have provided evidence that astrocytes play a role in sustaining tissue integrity in SCI [1,5,33-35]. However, activated astrocytes may take different phenotypes: A1, [3,4], which promotes an inflammatory environment detrimental to neuronal health, or A2 [3,4], is mainly said to suppress the pro-inflammatory milieu and regenerate tissue. This study demonstrated that PEG and PEI based-NG can be internalized in cells of the CNS, namely astrocytes and macrophages. NG designed with different moieties $\left(\mathrm{NH}_{2}, \mathrm{RhB}\right.$ or $\left.\mathrm{Cy} 5\right)$ changed the ability for internalization in these cells. $\mathrm{Cy} 5$ or $\mathrm{NH}_{2}+\mathrm{Cy} 5$ coating significantly reduced macrophage uptake. This demonstrates for the first time that common fluorochromes conjugated for tracking nanovectors can drive internalization into the cells. We showed particularly that mutually reinforcing can be established between the two groups $\left(\mathrm{NH}_{2}\right.$ and $\left.\mathrm{Cy} 5\right)$ functionalized on the surface of nanovectors, markedly suppressing NG uptake in these cells. We also noted a reduction of the internalization in astrocytes though less than microglia/macrophages. This indicates the interaction of two functional groups conjugated to NG limits the macrophage uptake machinery. Macrophages are crucial in immune surveillance and remove cell debris and foreign bodies from the organism [2,36]. Macrophages recognize nanovectors as foreign, and remove them from the environment [22], reducing the ability of the compound loaded in the nanovector to be properly targeted. Strategies to specifically avoid the uptake of nanostructures by macrophage populations are limited to some groups that reduce NP internalization. Conventional surface coating techniques enable PEG or recombinant CD47 protein to limit the phagocytic clearance of NPs [37]. In this study new surface modifications of NG were designed to avoid macrophage recognition so as to improve drug delivery and efficacy at the target.

Cell internalization is generally classified as either phagocytosis or pinocytosis. Evidence so far suggests four specific receptors capable of NP uptake: scavenger receptors, mannose receptors, Fc receptors and toll-like receptors [22]. NPs recognition and interaction with specific targets in macrophages is primarily influenced by surface charge, steric obstructions and protein adsorption on the surface (corona effect) [22]. The physicochemical characteristics of the functionalized groups together with the geometry and surface chemistry of the NG are capable of suppressing the internalization of NG in microglia/macrophage. This is likely ascribed to steric hindrance of the $\mathrm{NH}_{2}+\mathrm{Cy} 5$ groups inserted. These chemical groups change the hydrodynamic diameter from $155 \mathrm{~nm}$ to $215 \mathrm{~nm}$, but the charge is not changed. Alternately, $\mathrm{NH}_{2}+\mathrm{Cy} 5$ functionalization can cause a steric obstruction that reduces the corona effect, limiting phagocytic recognition.

In vitro, after treatment with $\mathrm{CPZ}$, the internalization of $\mathrm{NH} 2+\mathrm{Cy} 5$ NG is significantly reduced. This suggests that clathrin-dependent endocytosis could play a key role in the NG internalization, but other mechanisms could be involved. Once internalized in astrocytes, NG formulated with $\mathrm{NH}_{2}+\mathrm{Cy} 5$ were addressed to the lysosomal degradation pathway delivering PI, a molecule that can mimic the payload. This 


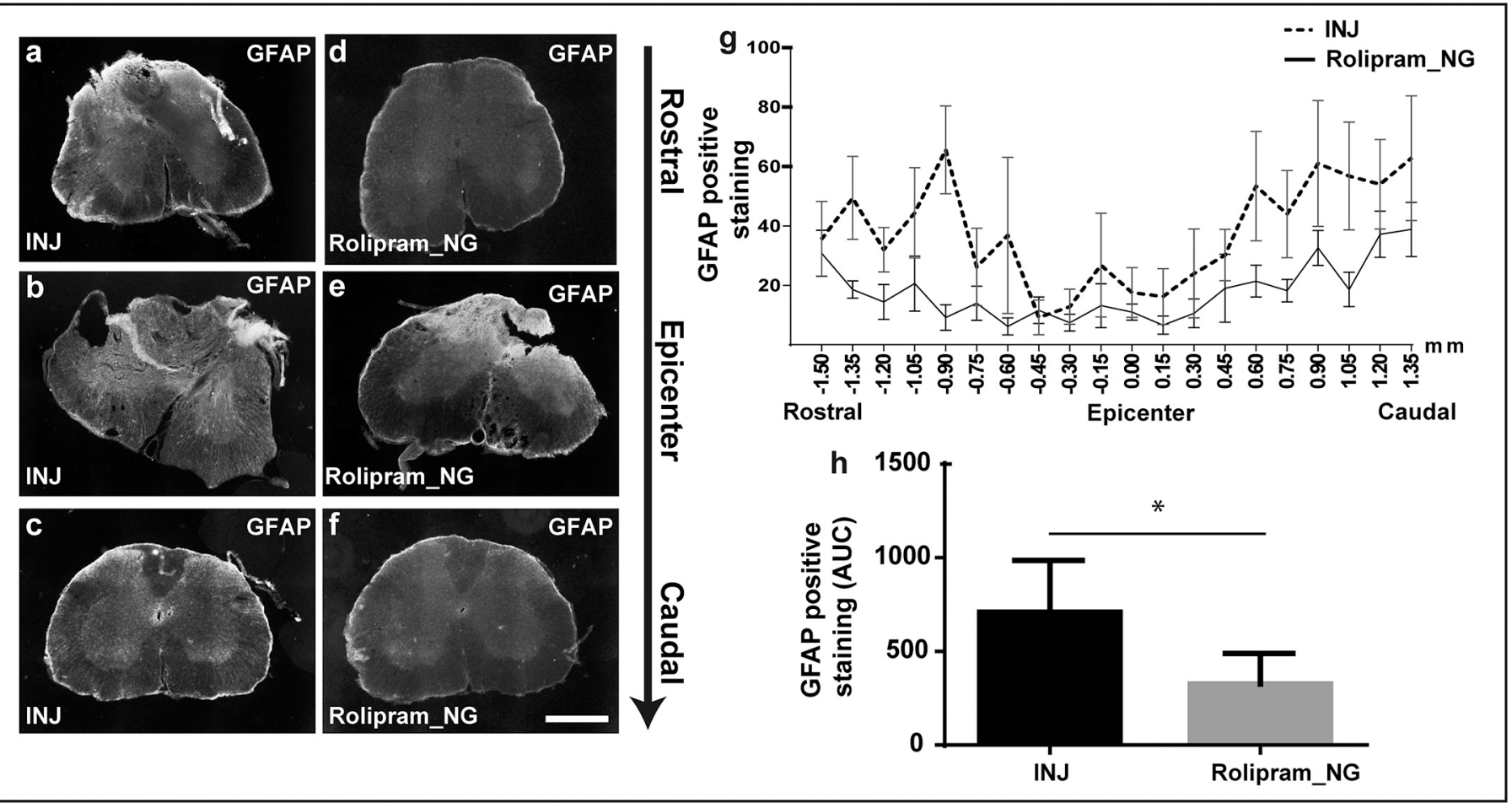

Fig. 7. Quantification of GFAP staining (g) in untreated injured mice (a, b, c, INJ) compared to the Rolipram-loaded NG (Rolipram_NG) treated group (d, e, f). There was a significant reduction of GFAP positive staining in treated mice (h). Data are mean \pm SD. Scale bar $400 \mu \mathrm{m}$. Mann-Whitney test. Statistical significance: $(* * * *) p$ $<0.0001 . N=5$ for each group.

indicates that the NG can be degraded in the astrocytes and release pharmacological compounds in vitro.

We also studied the uptake of NG formulations in astrocytes derived from human iPS cells, findings that the internalization of $\mathrm{NH}_{2}+\mathrm{Cy} 5$ functionalized NG was comparable with the uptake in murine astrocytes, suggesting the potential translatability to human patients.

In vitro results were validated by in vivo experiments: NG signal was distributed and colocalized almost exclusively with astrocytes for $\mathrm{NH}_{2}$ + Cy5 functionalized NG. Some microglia and neurons positive to NG were found only in the epicenter of the lesion. We can hypothesize that altered integrity of the membrane removes the controlled permeability of these cells.

The effect of this selective delivery system was demonstrated loading Rolipram in NG, and injecting $\mathrm{NH}_{2}+\mathrm{Cy} 5$ conjugated to NG into SCI mice. Rolipram inhibits NF-kB translocation from the cytosol to the nucleus, which in turn reduces the RNA transcription of proinflammatory molecules that can exacerbate the progression of the secondary injury [25]. Ex vivo analysis of the traumatized spinal cord indicated that the treatment reduced the GFAP positive staining. Recent research has described an astrogliosis mechanism based on a change of phenotype (hypertrophic) in neuroinflammatory and neurodegenerative conditions instead of cell proliferation [3]. So the efficacy of our treatment may be associated with the reduced hypertrophy of the cells rather than fewer astrocytes around the injured site, suggesting less proinflammatory reactivity in the tract of spinal cord reached by nanovectors.

\section{Conclusions}

Treatment of the inflammatory response in SCI remains a major clinical challenge. Targeting specific cells involved in this response may offer great advantage for improving the efficacy of treatment. Different NPs proposed to deliver therapeutics as an alternative to the viral vector treatment, have lacked selective action. Internalization of NPs by astrocytes was always complemented by microglia/macrophage or neuron uptake, limiting the therapeutic availability and increasing side effects.

This study demonstrated the ability of a new formulated NG to be almost exclusively internalized in astrocytes, limiting the phagocyte problem of macrophage/microglia. Therapeutic effects were also seen when loading an anti-inflammatory drug (Rolipram) able to act on the astrogliosis in an in vivo SCI model. Other molecules or compounds with potential therapeutic effect could be loaded by these nanovectors, opening up considerable promising for the treatment of the inflammatory response in SCI or other neurodegenerative diseases.

\section{Acknowledgements}

Authors' research is supported by Ministero della Salute (funding for an instrument purchase VS120 Olympus microscope) and Politecnico di Milano (nanovector characterization study).

\section{Appendix A. Supplementary data}

Supplementary data to this article can be found online at https://doi. org/10.1016/j.jconrel.2020.12.006.

\section{References}

[1] A.D. Gaudet, L.K. Fonken, Glial cells shape pathology and repair after spinal cord injury, Neurotherapeutics 15 (3) (2018) 554-577, https://doi.org/10.1007/ s13311-018-0630-7.

[2] X. Kong, J. Gao, Macrophage polarization: a key event in the secondary phase of acute spinal cord injury, J. Cell. Mol. Med. 21 (5) (2017) 941-954, https://doi.org/ 10.1111/jcmm.13034.

[3] S.A. Liddelow, B.A. Barres, Reactive astrocytes: production, Funct. Therap. Potent. Immun. 46 (6) (2017) 957-967, https://doi.org/10.1016/j.immuni.2017.06.006.

[4] S.A. Liddelow, K.A. Guttenplan, L.E. Clarke, F.C. Bennett, C.J. Bohlen, L. Schirmer, M.L. Bennett, A.E. Münch, W.-S. Chung, T.C. Peterson, D.K. Wilton, A. Frouin, B. A. Napier, N. Panicker, M. Kumar, M.S. Buckwalter, D.H. Rowitch, V.L. Dawson, T. M. Dawson, B. Stevens, B.A. Barres, Neurotoxic reactive astrocytes are induced by 
activated microglia, Nature 541 (7638) (2017) 481-487, https://doi.org/10.1038/ nature21029.

[5] M.A. Anderson, J.E. Burda, Y. Ren, Y. Ao, T.M. O'Shea, R. Kawaguchi, G. Coppola, B.S. Khakh, T.J. Deming, M.V. Sofroniew, Astrocyte scar formation aids central nervous system axon regeneration, Nature 532 (7598) (2016) 195-200, https:// doi.org/10.1038/nature17623.

[6] J.A. Smith, A. Braga, J. Verheyen, S. Basilico, S. Bandiera, C. Alfaro-Cervello, L. Peruzzotti-Jametti, D. Shu, F. Haque, P. Guo, S. Pluchino, RNA Nanotherapeutics for the amelioration of astroglial reactivity, Mol. Ther. Nucleic Acids 10 (2018) 103-121, https://doi.org/10.1016/j.omtn.2017.11.008.

[7] K. Gorshkov, F. Aguisanda, N. Thorne, W. Zheng, Astrocytes as targets for drug discovery, Drug Discov. Today 23 (3) (2018) 673-680, https://doi.org/10.1016/j drudis.2018.01.011.

[8] E.J. Bradbury, L.D.F. Moon, R.J. Popat, V.R. King, G.S. Bennett, P.N. Patel, J. W. Fawcett, S.B. McMahon, Chondroitinase ABC promotes functional recovery after spinal cord injury, Nature 416 (6881) (2002) 636-640, https://doi.org/ 10.1038/416636a.

[9] B.T. Lang, J.M. Cregg, M.A. DePaul, A.P. Tran, K. Xu, S.M. Dyck, K.M. Madalena, B. P. Brown, Y.-L. Weng, S. Li, S. Karimi-Abdolrezaee, S.A. Busch, Y. Shen, J. Silver, Modulation of the proteoglycan receptor PTP $\sigma$ promotes recovery after spinal cord injury, Nature 518 (7539) (2015) 404-408, https://doi.org/10.1038/nature13974.

[10] J.R. Siebert, A. Conta Steencken, D.J. Osterhout, Chondroitin sulfate proteoglycans in the nervous system: inhibitors to repair, Biomed. Res. Int. 2014 (2014) 845323, https://doi.org/10.1155/2014/845323.

[11] I. Vismara, S. Papa, F. Rossi, G. Forloni, P. Veglianese, Current options for cell therapy in spinal cord injury, Trends Mol. Med. 23 (9) (2017) 831-849, https:// doi.org/10.1016/j.molmed.2017.07.005.

[12] S. Papa, I. Caron, F. Rossi, P. Veglianese, Modulators of microglia: a patent review, Expert Opin. Ther. Pat. 26 (4) (2016) 427-437, https://doi.org/10.1517/ 13543776.2016.1135901.

[13] I. Caron, S. Papa, F. Rossi, G. Forloni, P. Veglianese, Nanovector-mediated drug delivery for spinal cord injury treatment, Wiley Interdiscip. Rev. Nanomed. Nanobiotechnol. 6 (5) (2014) 506-515, https://doi.org/10.1002/wnan.1276.

[14] F. Rossi, G. Perale, S. Papa, G. Forloni, P. Veglianese, Current options for drug delivery to the spinal cord, Expert Opin. Drug Deliv. 10 (3) (2013) 385-396, https://doi.org/10.1517/17425247.2013.751372.

[15] G. Perale, F. Rossi, E. Sundstrom, S. Bacchiega, M. Masi, G. Forloni, P. Veglianese, Hydrogels in spinal cord injury repair strategies, ACS Chem. Neurosci. 2 (7) (2011) 336-345, https://doi.org/10.1021/cn200030w.

[16] S. Papa, F. Rossi, I. Vismara, G. Forloni, P. Veglianese, Nanovector-mediated drug delivery in spinal cord injury: a multitarget approach, ACS Chem. Neurosci. 10 (3) (2019) 1173-1182, https://doi.org/10.1021/acschemneuro.8b00700.

[17] S. Papa, I. Caron, E. Erba, N. Panini, M. de Paola, A. Mariani, C. Colombo, R. Ferrari, D. Pozzer, E.R. Zanier, F. Pischiutta, J. Lucchetti, A. Bassi, G. Valentini, G. Simonutti, F. Rossi, D. Moscatelli, G. Forloni, P. Veglianese, Early modulation of pro-inflammatory microglia by minocycline loaded nanoparticles confers long lasting protection after spinal cord injury, Biomaterials 75 (2016) 13-24, https:// doi.org/10.1016/j.biomaterials.2015.10.015.

[18] S. Papa, R. Ferrari, M. de Paola, F. Rossi, A. Mariani, I. Caron, E. Sammali, M. Peviani, V. Dell'Oro, C. Colombo, M. Morbidelli, G. Forloni, G. Perale, D. Moscatelli, P. Veglianese, Polymeric nanoparticle system to target activated microglia/macrophages in spinal cord injury, J. Control. Release 174 (2014) 15-26, https://doi.org/10.1016/j.jconrel.2013.11.001.

[19] S. Papa, F. Rossi, R. Ferrari, A. Mariani, M. de Paola, I. Caron, F. Fiordaliso, C. Bisighini, E. Sammali, C. Colombo, M. Gobbi, M. Canovi, J. Lucchetti, M. Peviani, M. Morbidelli, G. Forloni, G. Perale, D. Moscatelli, P. Veglianese, Selective nanovector mediated treatment of activated proinflammatory microglia/ macrophages in spinal cord injury, ACS Nano 7 (11) (2013) 9881-9895, https:// doi.org/10.1021/nn4036014.

[20] W. Gao, R.B. Borgens, Remote-controlled eradication of astrogliosis in spinal cord injury via electromagnetically-induced dexamethasone release from "smart" nanowires, J. Control. Release 211 (2015) 22-27, https://doi.org/10.1016/j. jconrel.2015.05.266.

[21] X. Li, K. Kozielski, Y.-H. Cheng, H. Liu, C.G. Zamboni, J. Green, H.-Q. Mao, Nanoparticle-mediated conversion of primary human astrocytes into neurons and oligodendrocytes, Biomater. Sci. 4 (7) (2016) 1100-1112, https://doi.org/ 10.1039/c6bm00140h.

[22] H.H. Gustafson, D. Holt-Casper, D.W. Grainger, H. Ghandehari, Nanoparticle uptake: the phagocyte problem, Nano Today 10 (4) (2015) 487-510, https://doi. org/10.1016/j.nantod.2015.06.006.

[23] H. Zhang, Y. Zhai, J. Wang, G. Zhai, New progress and prospects: the application of nanogel in drug delivery, Mater. Sci. Eng. C Mater. Biol. Appl. 60 (2016) 560-568, https://doi.org/10.1016/j.msec.2015.11.041.

[24] E. Mauri, P. Veglianese, S. Papa, A. Rossetti, M. de Paola, A. Mariani, Z. Posel, P. Posocco, A. Sacchetti, F. Rossi, Effects of primary amine-based coatings on microglia internalization of nanogels, Colloids Surf. B: Biointerfaces 185 (2020) 110574, https://doi.org/10.1016/j.colsurfb.2019.110574.

[25] R. Hervé, T. Schmitz, D. Evain-Brion, D. Cabrol, M.-J. Leroy, C. Méhats, The PDE4 inhibitor rolipram prevents NF-kappaB binding activity and proinflammatory cytokine release in human chorionic cells, J. Immunol. 181 (3) (2008) 2196-2202, https://doi.org/10.4049/jimmunol.181.3.2196.

[26] E. Mauri, P. Veglianese, S. Papa, A. Mariani, M. de Paola, R. Rigamonti, G.M. F. Chincarini, S. Rimondo, A. Sacchetti, F. Rossi, Chemoselective functionalization of nanogels for microglia treatment, Eur. Polym. J. 94 (2017) 143-151, https:// doi.org/10.1016/j.eurpolymj.2017.07.003.

[27] E. Mauri, I. Moroni, L. Magagnin, M. Masi, A. Sacchetti, F. Rossi, Comparison between two different click strategies to synthesize fluorescent nanogels for therapeutic applications, React. Funct. Polym. 105 (2016) 35-44, https://doi.org/ 10.1016/j.reactfunctpolym.2016.05.007.

[28] I. Vismara, S. Papa, V. Veneruso, E. Mauri, A. Mariani, M. de Paola, R. Affatato, A. Rossetti, M. Sponchioni, D. Moscatelli, A. Sacchetti, F. Rossi, G. Forloni, P. Veglianese, Selective modulation of A1 astrocytes by drug-loaded Nanostructured gel in spinal cord injury, ACS Nano 14 (1) (2020) 360-371, https://doi. org/10.1021/acsnano.9b05579.

[29] M. de Paola, V. Diana, P. Bigini, T. Mennini, Morphological features and responses to AMPA receptor-mediated excitotoxicity of mouse motor neurons: comparison in purified, mixed anterior horn or motor neuron/glia cocultures, J. Neurosci.

Methods 170 (1) (2008) 85-95, https://doi.org/10.1016/j.jneumeth.2007.12.022.

[30] Y. Yan, S. Shin, B.S. Jha, Q. Liu, J. Sheng, F. Li, M. Zhan, J. Davis, K. Bharti, X. Zeng, M. Rao, N. Malik, M.C. Vemuri, Efficient and rapid derivation of primitive neural stem cells and generation of brain subtype neurons from human pluripotent stem cells, Stem Cells Transl. Med. 2 (11) (2013) 862-870, https://doi.org/ 10.5966/sctm.2013-0080.

[31] L.M. Cruz-Orive, E.R. Weibel, Recent stereological methods for cell biology: a brief survey, Am. J. Phys. 258 (4 Pt 1) (1990) L148-L156, https://doi.org/10.1152/ ajplung.1990.258.4.L148.

[32] H.J. Gundersen, E.B. Jensen, The efficiency of systematic sampling in stereology and its prediction, J. Microsc. 147 (Pt 3) (1987) 229-263.

[33] M.V. Sofroniew, Dissecting spinal cord regeneration, Nature 557 (7705) (2018) 343-350, https://doi.org/10.1038/s41586-018-0068-4.

[34] M.B. Orr, J.C. Gensel, Spinal cord injury scarring and inflammation: therapies targeting glial and inflammatory responses, Neurotherapeutics 15 (3) (2018) 541-553, https://doi.org/10.1007/s13311-018-0631-6.

[35] S.A. Liddelow, B.A. Barres, Regeneration: not everything is scary about a glial scar, Nature 532 (7598) (2016) 182-183, https://doi.org/10.1038/nature17318.

[36] A. Kroner, J. Rosas Almanza, Role of microglia in spinal cord injury, Neurosci. Lett. 709 (2019) 134370, https://doi.org/10.1016/j.neulet.2019.134370.

[37] Y. Qie, H. Yuan, C.A. von Roemeling, Y. Chen, X. Liu, K.D. Shih, J.A. Knight, H. W. Tun, R.E. Wharen, W. Jiang, B.Y.S. Kim, Surface modification of nanoparticles enables selective evasion of phagocytic clearance by distinct macrophage phenotypes, Sci. Rep. 6 (2016) 26269, https://doi.org/10.1038/srep26269. 